\title{
Effect of Topical Propolis on Wound Healing Process After Tonsillectomy: Randomized Controlled Study
}

\author{
Jeong Hwan Moon ${ }^{1-4} \cdot$ Min Young Lee ${ }^{1,4} \cdot$ Young-Jun Chung ${ }^{1,4} \cdot$ Chung-Ku Rhee ${ }^{1,2} \cdot$ Sang Joon Lee ${ }^{1,3,4}$ \\ ${ }^{1}$ Department of Otolaryngology-Head and Neck Surgery, Dankook University College of Medicine, Cheonan; ${ }^{2}$ Medical Laser Research Center, \\ ${ }^{3}$ Beckman Laser Institute Korea, and ${ }^{4}$ Laser Translational Clinical Trial Center, Dankook University, Cheonan, Korea
}

Objectives. The post-tonsillectomy pain and post-tonsillectomy hemorrhage are the two main problems after tonsillectomy. The aim of this study was to investigate the beneficial effects of water soluble ethanol extract propolis on post-tonsillectomy patient.

Methods. One hundred and thirty patients who underwent tonsillectomy or adenotonsillectomy were randomly divided into the control and propolis groups, each including 65 patients. The propolis group was applied with propolis orally immediately after surgery and by gargle. The pain scores were assessed on post-tonsillectomy $0,1 \mathrm{st}$, 2nd, 3rd, and 7th-10th day using a visual analogue scale score. Postoperative wound healing was evaluated by scoring pinkish membrane of tonsillar fossae on postoperative days 3 and $7-10$. The incidence of post-tonsillectomy bleeding was examined in each group.

Results. Post-tonsillectomy pain was significantly less in propolis group compared to control group on postoperative days 3 and 7-10. Post-tonsillectomy hemorrhage was significantly less in the propolis group compared to the control group $(P<0.05)$. The wound healing was significantly better in the propolis group compared to the control group on postoperative day $7-10(P=0.002)$.

Conclusion. Applying the propolis to post-tonsillectomy wound showed beneficial effect of reducing postoperative pain, preventing hemorrhage, and accelerating of wound healing of tonsillar fossae.

Keywords. Propolis; Tonsillectomy; Pain; Hemorrhage; Wound Healing

\section{INTRODUCTION}

Tonsillectomy is one of common surgical procedures performed in otolaryngology. Recurrent tonsillitis, obstructive sleep apnea, mouth breathing, recurrent otitis media, swallowing or respiratory difficulty due to tonsillar hypertrophy, and peritonsillar abscess are the indications for this procedure. Complications after tonsillectomy are hemorrhage, pain, airway obstruction, pharyngeal insufficiency, and pulmonary edema. Among these complication,

\footnotetext{
- Received June 2, 2017

Revised August 24, 2017

Accepted September 27, 2017

- Corresponding author: Sang Joon Lee

Department of Otolaryngology-Head and Neck Surgery, Dankook

University College of Medicine, 201 Manghyang-ro, Dongnam-gu,

Cheonan 31116, Korea

Tel: +82-41-550-1780, Fax: +82-41-550-1090

E-mail: 1sj72@dankook.ac.kr
}

post-tonsillectomy pain and hemorrhage are still major problems.

Propolis is a resinous mixture that honey bees collect from tree buds, sap flows, or other botanical sources. The composition of propolis varies from hive to hive, district to district, and from season to season. The major component of propolis is flavonoid and the other minor components are phenol acids, alcohol-aldehydes, coumarins, vitamins, and minerals. Water soluble ethanol extract propolis (WEEP; Seoul Propolis Co., Daejeon, Korea) is a nontoxic natural product with multiple pharmacological effects including anti-inflammatory, tissue regenerative, antibacterial, antifungal, antiviral, antioxidant, and cicatrization properties. Three distinct chemical groups have been reported to be present: (1) flavonoid aglycones, (2) cinnamic acid derivatives, and (3) terpenoids. Flavonoids have been considered the main biologically active compounds [1-3]. Major component causing antioxidant and cicatrization effect is suspected as flavonoid [3-6].

These various effects of propolis could induce rapid wound

Copyright $\odot 2018$ by Korean Society of Otorhinolaryngology-Head and Neck Surgery.

This is an open-access article distributed under the terms of the Creative Commons Attribution Non-Commercial License (http://creativecommons.org/licenses/by-nc/4.0)

which permits unrestricted non-commercial use, distribution, and reproduction in any medium, provided the original work is properly cited. 
healing, less pain, and decreased postoperative hemorrhage. The earlier they heal, the better the patients would feel with less pain, less chances of hemorrhage, and earlier return to works. This study was designed to investigate the beneficial effects of WEEP on tonsillectomy patients.

\section{MATERIALS AND METHODS}

One hundred fifty-five patients underwent tonsillectomy or adenotonsillectomy from December 2011 to April 2012 at the Department of Otorhinolaryngology of Dankook University Hospital. Indications of tonsillectomy or adenotonsillectomy were snoring, recurrent tonsillitis, chronic hypertrophic tonsillitis, and peritonsillar abscess. The patients with tonsillectomy or adenotonsillectomy combined with septoplasty, palatoplasty, and other operations were excluded.

This study was designed as balanced randomization (1:1), placebo-controlled, parallel-group study. Eligible participants were 130 patients. Patients were randomly assigned following simple randomization to one of two groups, control and propolis groups, and each contained 65 patients. Flow diagram of the study is shown in Fig. 1. The day before each operation, we explained the objectives of this study and visual analogue scale (VAS) scoring system to patients and written informed consent was obtained. Tonsillectomy or adenotonsillectomy was performed under general anesthesia. There was no difference of surgical procedures and surgeon difference between two groups. In the present study, two professional ENT specialist who are the authors of the manuscript (YJC, SJL) has performed the operation, and these surgeons used consistent surgical techniques throughout the study. Bleeding was controlled by electrocauterization. The propolis group was applied with topical propolis gel (Seoul Propolis Co.) on bilateral tonsil fossae immediately after tonsillectomy procedure. Both groups were administered with Cefazedone (Cefazedone sodium $1 \mathrm{~g}$; Kookje Chemical Co., Hwaseong, Korea) as an antibiotics and Ultracet (acetaminophen $325 \mathrm{mg}+$ tramadol $37.5 \mathrm{mg}$; Janssen, Seoul, Korea) as an analgesics for 3 days after the operation. WEEP bottles (10

\section{H I G H L I G H T S}

- This study is a prospective randomized trial of the effect of propolis on wound healing process after tonsillectomy.

- Post-tonsillectomy pain was significantly less in propolis group compared to control group on postoperative days 3 and 7-10.

- The wound healing was significantly better in the propolis group compared to the control group on postoperative day 7-10.

- Post-tonsillectomy hemorrhage was significantly less in the propolis group compared to the control group.

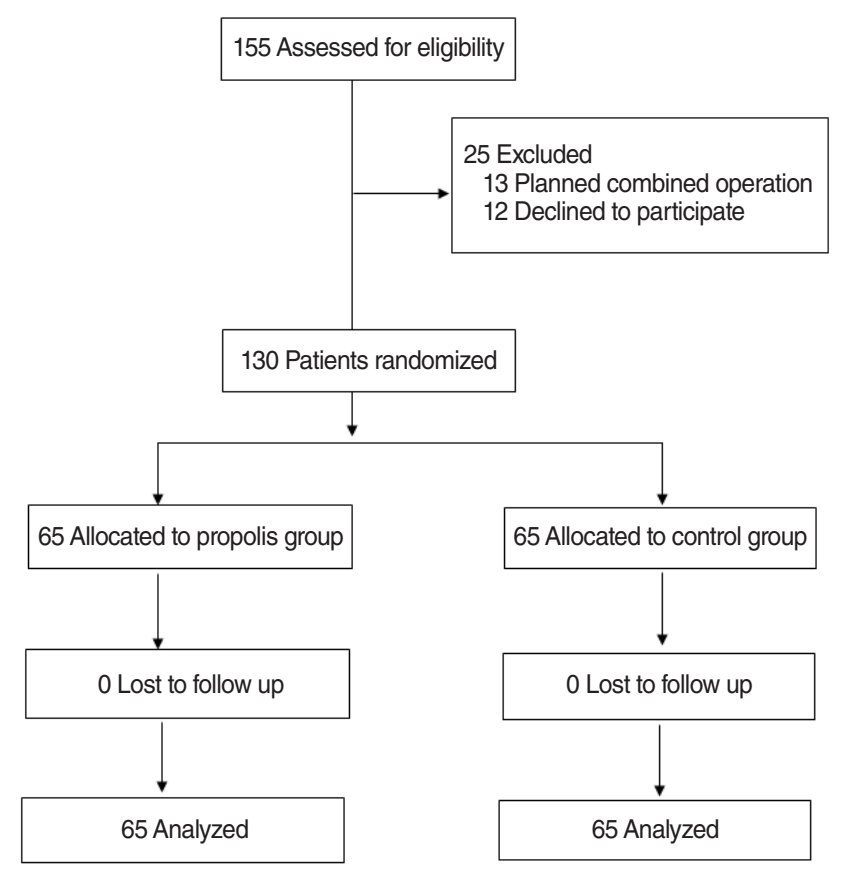

Fig. 1. Flow diagram of propolis application.

drops of WEEP mixed in a glass of $150 \mathrm{~mL}$ water) were provided to the patients in propolis group and placebo bottles which contained similar WEEP colored liquid which had very similar taste to WEEP bottle were provided to patients in control group. The composition of placebo bottle fluid is coloring matter for food. Patients in both groups gargled throat and swallowed with eitherWEEP or placebo four times per day. After discharge from the hospital, Tylenol $650 \mathrm{mg}$ (acetaminophen, Janssen) was administered orally for control of pain for 7 days.

All patients recorded pain levels on post-tonsillectomy 0,1 st, 2nd, 3rd, and 7th-10th day (first postoperative visit to clinic) using a VAS. VAS point 0 meant no pain, and point 10 indicated most severe pain.

Postoperative wound healing was evaluated by scoring the size of pinkish membrane coverage over the tonsillar fossae [7] on postoperative days 3 and 7-10. In the present study, surgeons (the two co-authors who did the tonsillectomy) observed the pain, hemorrhage, and wound healing scores and they were blind to the randomization. If proportion of pinkish area on tonsillar fossa is below $10 \%$, score on wound healing was 0 point; 1 point was from $10 \%$ to $25 \%$; 2 point was from $25 \%$ to $50 \%$; and 3 point was above $50 \%$ (Fig. 2). The incidence of post-tonsillectomy hemorrhage was examined in each group. Each patient was instructed to visit emergency room of the tertiary university hospital if tonsillar bleeding develops.

\section{Ethical considerations}

This study has been approved by the Ethical Committee and Institutional Review Board of Faculty of Medicine, Dankook Uni- 

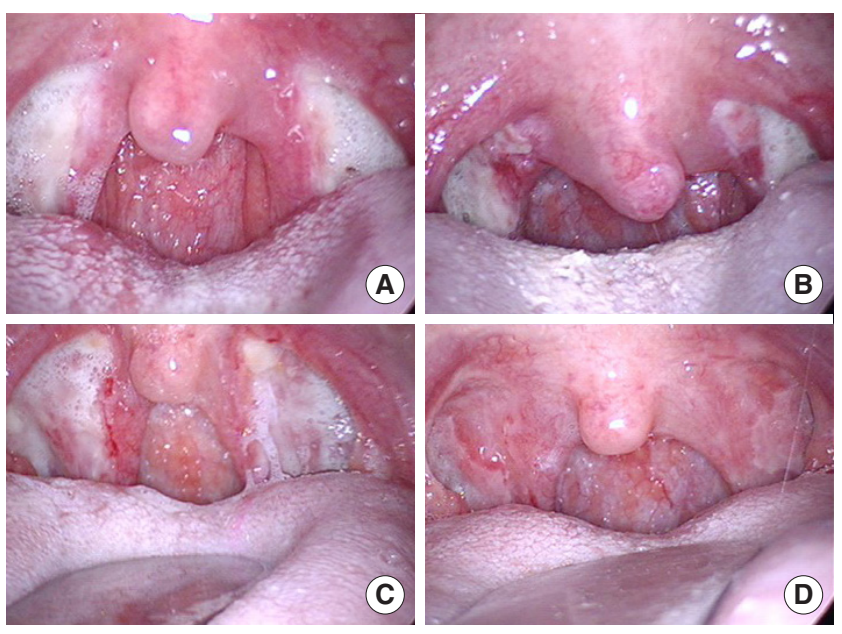

Fig. 2. The representative images of wound healing process: point 0 $(A)$, point $1(B)$, point $2(C)$, and point $3(D)$.

Table 1. Demographic characteristics of the control and propolis groups according to sex and age

\begin{tabular}{lccc}
\hline Characteristic & $\begin{array}{c}\text { Control group } \\
(n=65)\end{array}$ & $\begin{array}{c}\text { Propolis group } \\
(n=65)\end{array}$ & $P$-value \\
\hline Sex (male:female) & $40: 25$ & $41: 24$ & 0.856 \\
Age (yr) & $16.1 \pm 12.8$ & $13.9 \pm 12.4$ & 0.322 \\
\hline
\end{tabular}

Values are presented as mean \pm standard deviation.

versity Hospital (IRB No. 1021-103). Written informed consent was obtained in each patient before study enrollment.

\section{Statistical analyses}

Each value was shown by mean \pm two standard deviations. The $t$-test was performed for VAS score of pain and wound healing. Wound healing and hemorrhage were evaluated by chi-square analysis and Cochran-Armitage test. Statistical significance was set at $P<0.05$.

\section{RESULTS}

The average age was $16.1 \pm 12.8$ years for control and $13.9 \pm$ 12.4 years for propolis groups (Table 1 ). There was no statistical age difference between the two groups $(P=0.322)$. In control group, average of VAS score of post-tonsillectomy pain was 5.63 on day $0,5.51$ on day $1,5.2$ on day $2,4.94$ on day 3 , and 3.72 on day 7-10 postoperatively. In propolis group, average of VAS score of post-tonsillectomy pain was 5.75 on day $0,5.42$ on day $1,4.85$ on day $2,4.25$ on day 3 , and 2.97 on day 7-10 postoperatively.VAS score was shown downward trend with or without propolis. Post-tonsillectomy pain was significantly less in propolis group compared to control group on postoperative days 3 and 7-10 (Fig. 3).

In control group, the number of patients at wound healing

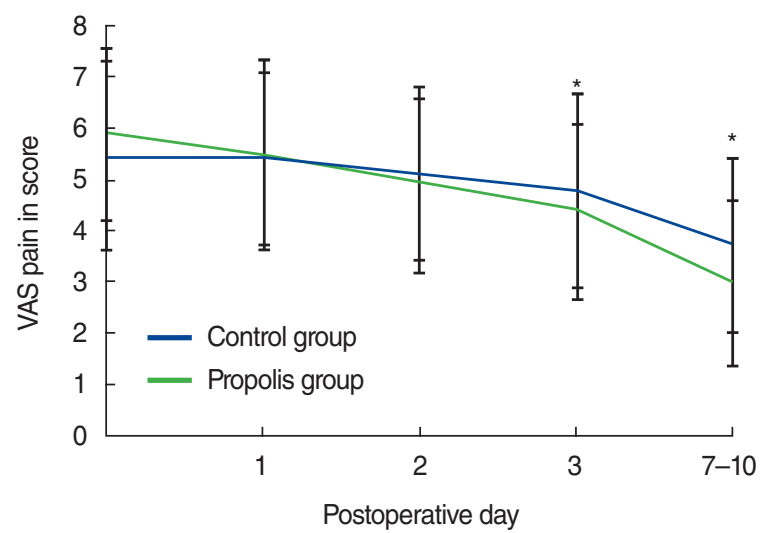

Fig. 3. Visual analogue scale (VAS) pain score. Pain scores were determined by a VAS on post-tonsillectomy 0 , 1st, 2nd, 3rd, and 7th10th day. The VAS score on postoperative days 3 and $7-10$ was significantly different between the two groups $\left({ }^{\star} P<0.05\right)$.

score 0 was 29 and 36 at 1 point on postoperative day 3. On postoperative day $7-10$, number of patients was five at 0 point, 30 at 1 point, 24 at 2 point, and six at 3 point. In propolis group, the number of patients at wound healing score 0 point was 33 and 32 at 1 point on postoperative day 3 . On postoperative day $7-10$, the number of patients at 0 point was two, 11 at 1 point, 38 at 2 point, and 14 at 3 point. The patient proportion of high scores (scores 2 and 3 ) was higher in propolis group compared to control group on postoperative day 7-10 $(P<0.001$, Linearby-Linear association value $=13.580, d f=1)($ Fig. $4 \mathrm{~A})$ and the mean score of wound healing was higher in propolis group compared to the control group on postoperative day 7-10 $(P=0.002$, $t=3.381, d f=128$ ) (Fig. 4B).

Post-tonsillectomy hemorrhage was developed in 14 patients among 130 patients. Average age of the patients with bleeding was 20.6 years. Male to female ratio was $5: 2$. It has occurred more frequently in male. Hemorrhage was occurred on 7.71 postoperative days. There was no postoperative bleeding within 3rd postoperative day. In the control group, frequency of hemorrhage was $16.9 \%, 11$ of 65 patients. In propolis group, frequency of hemorrhage was $4.6 \%$, three of 65 patients. Post-tonsillectomy hemorrhage was significantly less in propolis group compared to that of control group $(P<0.05)$. Among 14 post-tonsillectomy hemorrhage patients of control group, recurrent hemorrhage which requires bleeding control under general anesthesia was encountered to four patients. In propolis group, recurrent hemorrhage was not observed and they were discharged without any further procedure.

\section{DISCUSSION}

Post-tonsillectomy pain is due to hyperalgesia and nerve regeneration on damaged area. Hyperalgesia at the site of an injury can be explained by sensitization of nociceptors. This sensitiza- 

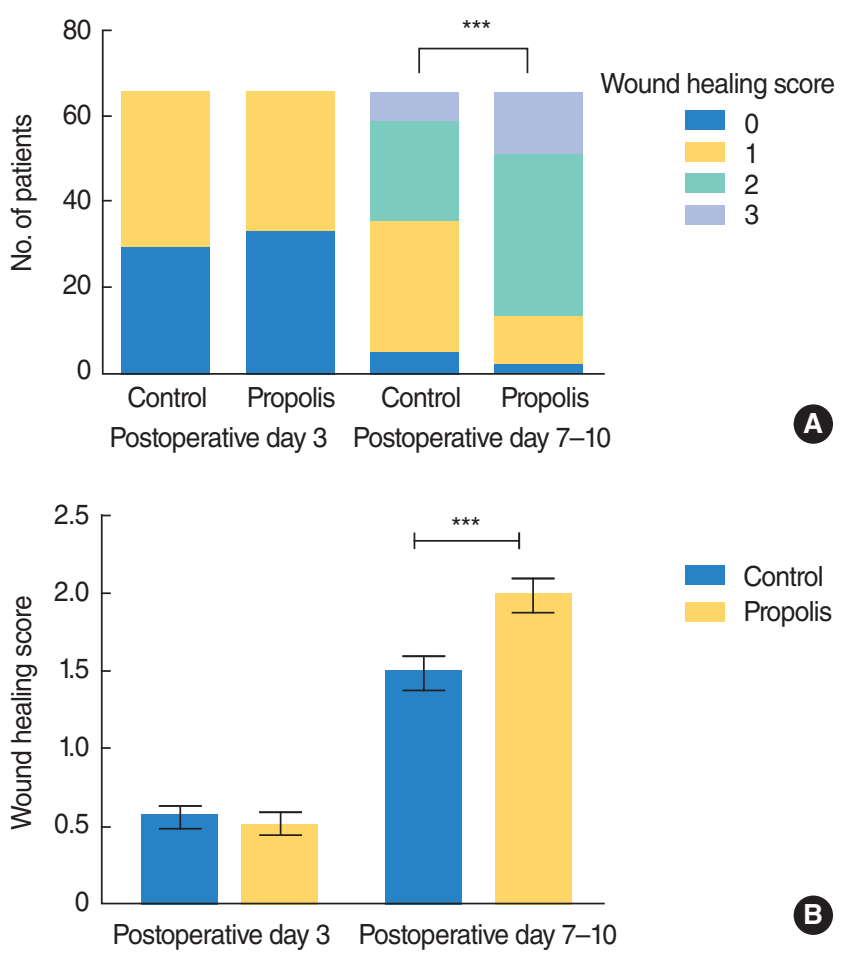

Fig. 4. Wound healing score. Wound healing scores evaluated by presence of pinkish membrane at tonsillar fossae on postoperative days 3 and 7-10. (A) The patient proportion of high scores (scores 2 and 3 ) was higher in propolis group compared to control group on postoperative day $7-10$ while it was not significant on postoperative day $3\left({ }^{* \star *} P<0.001\right)$. (B) The mean score of wound healing was higher in propolis group compared with the control group on postoperative day $7-10$ while it was not significant on postoperative day 3. ${ }^{\star \star \star} P=0.002$.

tion is likely due to local release of chemical mediators in inflamed tissue, that decreases the threshold of transient receptor potential vanilloid 1 on A $\delta$-fibers and C-fibers of nerves [8]. Opioids, non-steroidal anti-inflammatory drugs, and topical analgesics spray or infiltration has been suggested to improve postoperative pain relief. In this study, post-tonsillectomy pain was significantly reduced in the propolis group compared to the control group on postoperative days 3 and 7-10, suggesting the postoperative pain relief effect of propolis similar to other materials addressed above. There are no previous report addressing this issue but as to aphthous ulcer propolis has shown effective pain control [5]. This effect could be explained by anti-inflammatory activity through inhibiting the release of arachidonic acid from cell membrane, which leads to the suppression of cyclooxygenase (COX)-1 and COX-2 activities [6]. Flavonoid, caffeic acid phenethyl ester, which present in propolis, is thought to have this anti-inflammatory effect. Alternatively, post-tonsillectomy pain relieving might be associated with the decrease of reactive oxygen species, which is thought to be related to the pain mechanism [9], by main components of propolis that have antioxidant activity [6].
It is reasonable to believe that propolis was effective for enhancing the wound healing based on current study. The degree of wound healing has been measured by the size of healing membrane after tonsillectomy, and the wound healing was significantly better in the propolis group compared to the control group on the first outpatient department visit to clinic. Propolis is known to enhance tissue regeneration and scar formation. When propolis is applied to a wound site with open epithelium, propolis infiltrates deeply into the tissue, stimulates cell regeneration, increases the proliferation of cornification cell, and attributes to wound healing [6]. In burn wound, compared to silver sulfadiazine that is commonly used to treat burn wound, propolis was the more useful in wound healing [7]. Flavonoid in propolis presents cicatrization effect by preventing of acid secretion, increasing prostaglandin concentration, inhibiting lipid peroxidation, and decreasing reactive oxygen species, and these processes would eventually facilitate the epithelial regeneration and scar formation $[4,6]$.

The most frequent and serious complication of tonsillectomy is post-tonsillectomy hemorrhage, which occurs at a rate about $5 \%$ (between $0.28 \%$ and $20 \%$ ) [10-12]. Primary hemorrhage is defined as bleeding that occurs in the first 24 postoperative hours [12]. Cause of primary hemorrhage is mostly failure of coagulation, ligation, and cauterization. Secondary hemorrhage, or postoperative bleeding after 24 hours [12], is due to sloughing of eschar, secondary trauma to solid food ingestion, tonsil bed infection, thermal damage on operation field, and postoperative nonsteroidal anti-inflammatory drug usage. Post-tonsillectomy hemorrhage is a fatal complication in some cases. Intensive care could be required such as fluid therapy, cross matching of blood types, ligation of blood vessels, compression of tonsillar fossa, and electro cauterization. Only four of 65 patients in control group had to be taken to the operating room for the control of hemorrhage and all patients in propolis group were released from the hospital only after observation without further procedures. Total number of postoperative hemorrhage in this study was relatively high since even very small amount of hemorrhage was included as post-tonsillectomy hemorrhage.

In the present study, control substance was only used for gargle not for the gel sheets after the surgery. It is less likely that the gel sheet coating without propolis would prevent the nonimmediate bleeding or pain scores. However, immediate pain scores and bleeding rate might be affected by the existence of gel sheeting after the surgery. For more objective analysis of the effect of propolis on tonsillectomy patients, controlled study for the gel sheeting might be necessary. Administration of propolis gel and WEEP to post-tonsillectomy patients appeared to be beneficial on postoperative pain control, preventing hemorrhage, and acceleration of wound healing in tonsillar fossae. Clinically, administration of propolis seems to be worthwhile, considering its beneficial effects on postoperative complications and wound healing. 


\section{CONFLICT OF INTEREST}

No potential conflict of interest relevant to this article was reported.

\section{ACKNOWLEDGMENTS}

This research was supported by a grant of the Korea Health Technology R\&D Project through the Korea Health Industry Development Institute (KHIDI), funded by the Ministry of Health \& Welfare (No. HI14C1234), Republic of Korea.

\section{REFERENCES}

1. Magro Filho O, de Carvalho AC. Application of propolis to dental sockets and skin wounds. J Nihon Univ Sch Dent. 1990 Mar;32(1): 4-13.

2. Velikova M, Bankova V, Marcucci MC, Tsvetkova I, Kujumgiev A. Chemical composition and biological activity of propolis from Brazilian meliponinae. Z Naturforsch C. 2000 Sep-Oct;55(9-10):785-9.

3. Khayyal MT, el-Ghazaly MA, el-Khatib AS, Hatem AM, de Vries PJ, el-Shafei S, et al. A clinical pharmacological study of the potential beneficial effects of a propolis food product as an adjuvant in asthmatic patients. Fundam Clin Pharmacol. 2003 Feb;17(1):93-102.

4. Martin MJ, La-Casa C, Alarcon-de-la-Lastra C, Cabeza J, Villegas I, Motilva V.Anti-oxidant mechanisms involved in gastroprotective effects of quercetin. Z Naturforsch C. 1998 Jan-Feb;53(1-2):82-8.

5. Samet N, Laurent C, Susarla SM, Samet-Rubinsteen N. The effect of bee propolis on recurrent aphthous stomatitis: a pilot study. Clin Oral Investig. 2007 Jun;11(2):143-7.

6. Viuda-Martos M, Ruiz-Navajas Y, Fernandez-Lopez J, Perez-Alvarez JA. Functional properties of honey, propolis, and royal jelly. J Food Sci. 2008 Nov;73(9):R117-24.

7. Akbas Y, Pata YS, Unal M, Gorur K, Micozkadioglu D. The effect of fusafungine on post-operative pain and wound healing after pediatric tonsillectomy. Int J Pediatr Otorhinolaryngol. 2004 Aug;68(8): 1023-6.

8. Raja SN, Meyer RA, Campbell JN. Peripheral mechanisms of somatic pain. Anesthesiology. 1988 Apr;68(4):571-90.

9. Ibi M, Matsuno K, Shiba D, Katsuyama M, Iwata K, Kakehi T, et al. Reactive oxygen species derived from NOX1/NADPH oxidase enhance inflammatory pain. J Neurosci. 2008 Sep;28(38):9486-94.

10. Szeremeta W, Novelly NJ, Benninger M. Postoperative bleeding in tonsillectomy patients. Ear NoseThroat J. 1996 Jun;75(6):373-6.

11. WeimertTA, Babyak JW, Richter HJ. Electrodissection tonsillectomy. Arch Otolaryngol Head Neck Surg. 1990 Feb;116(2):186-8.

12. Perkins JN, Liang C, Gao D, Shultz L, Friedman NR. Risk of posttonsillectomy hemorrhage by clinical diagnosis. Laryngoscope. 2012 Oct;122(10):2311-5. 